\title{
A Report of Nausea and Vomiting with Discontinuation of Chronic Use of Salvia divinorum
}

\author{
C. R. Travis, ${ }^{1}$ G. A. Ray, ${ }^{1}$ and K. F. Marlowe ${ }^{1,2}$ \\ ${ }^{1}$ Department of Pharmacy Practice, Harrison School of Pharmacy, Auburn University, Auburn, AL 36849, USA \\ ${ }^{2}$ Department of Internal Medicine, University of South Alabama, 650 Health Services Building, Suite 2100, Mobile, AL 36688, USA
}

Correspondence should be addressed to K. F. Marlowe, marlokf@auburn.edu

Received 1 July 2011; Accepted 5 January 2012

Academic Editor: Yedidia Bentur

Copyright ( 2012 C. R. Travis et al. This is an open access article distributed under the Creative Commons Attribution License, which permits unrestricted use, distribution, and reproduction in any medium, provided the original work is properly cited.

\begin{abstract}
Introduction. This is the first reported case of gastrointestinal symptoms associated with withdrawal after chronic use of this substance. Case Presentation. A 51-year-old Caucasian woman was referred to a hospital with a 3-day history of nausea, vomiting, diarrhea, and abdominal discomfort. She reported no sick family members or contact with anyone who was ill. She did report smoking 3-5 cigarettes of the herb "Salvia" consistently for 3-4 months and quit approximately 48 hours before symptoms appeared. Her use of the herb had been consistent; she smoked several cigarettes each day. Laboratory results were essentially normal including the white blood cell count. She received symptomatic treatment and was released after one day. Discussion. Salvinorin A, a kappa-opioid receptor agonist, is the major active ingredient of S. divinorum. The unique opioid properties of this herb may explain its ability to cause changes in intestinal transit time. Conclusion. A 51-year-old woman possibly developed gastrointestinal manifestations suggestive of withdrawal from Salvia divinorum after smoking the substance consistently for 3 to 4 months. The widespread use of this herb will make the potential for withdrawal syndromes more commonplace.
\end{abstract}

\section{Introduction}

Although its possession and use is currently illegal in more than 13 nations and 15 United States (US) states, Salvia divinorum use remains widespread [1]. In a 2008 survey, an estimated 1.8 million people in the US had tried this herb [2]. S. divinorum, a member of the mint family, is a commonly abused herb because of its associated hallucinogenic effects $[1,3-6]$. Use of this substance dates back several centuries for spiritual practices by the Mazatec Indians of Mexico [1, 37]. Other names for this herb include "Diviner's Sage" and "Magic Mint." Adolescents and young adults are particularly attracted to S. divinorum because of its accessibility, legality in some areas, pereception of relative safety, and lack of detectability upon urine drug screening [3]. Inhalation of S. divinorum is most commonly done by smoking the dried leaves or leaf extracts. Other ways of administration include inhalation via volatilization, or using tinctures for buccal administration $[1,3,5]$.

Salvinorin A, a kappa-opioid receptor agonist, has been demonstrated to be the major active ingredient of
S. divinorum [1,3-6]. Taken orally, Salvinorin A has a mild effect. It is only minimally absorbed through the mouth while most is degraded in the gastrointestinal tract [3]. When smoked, however, this compound's effects are much more pronounced generating hallucinations within seconds that may last from 30 minutes to over 2 hours depending on the amount inhaled $[4,6]$. Salvinorin A is currently the most potent naturally occurring hallucinogen to be isolated [4]. Due to its hallucinogenic effects and lack of evidence for medicinal purposes, nine other US states currently have Salvia divinorum or Salvinorin A labeled as a Schedule 1 substance. As a Schedule 1 substance, this agent has high potential for abuse, no proven medicinal use, and is potentially unsafe [8].

The most commonly reported desired effects of $S$. divinorum include the "trip," laughter, happiness, separation from body, relaxation, visual and auditory effects, perceptual modification, and pleasant after effects $[3,5]$. Loss of consciousness has also been reported with this herb [9]. Unpleasant after effects include reports of tiredness, heaviness of head, dizziness, physical exhaustion, and slowed 
mental function [5]. However, a search of the medical literature failed to reveal any case reports of a $S$. divinorum withdrawal syndrome. We report a case of $S$. divinorum withdrawal in a patient who presents to the emergency department with gastrointestinal distress after discontinuing use of cigarettes containing the herb. One previous series of patients with exposures to Salvia divinorum included one patient with gastrointestinal symptoms similar to those presented in our case. To our knowledge, this is the first case report addressing $S$. divinorum withdrawal.

\section{Case Report}

A 51-year-old Caucasian woman with hypertension, chronic obstructive pulmonary disease, coronary artery disease, hypothyroidism, and type 2 diabetes was referred to the hospital in June 2010 following a clinic visit for nausea, vomiting, diarrhea, and abdominal discomfort. Surgical history includes a coronary artery bypass graft (CABG) involving aortic and pulmonic valve replacements, hysterectomy, cholecystectomy, and an appendectomy. The patient reports smoking cigarettes (2 packs/day) and occasional alcohol use, but reports no other comorbidities or previous episodes involving these symptoms. She denies abdominal pain associated with food or bowel movements, or any melena, hematochezia, hematuria, or hemoptysis. Patient reports eating nothing out of the ordinary at home and not eating out during the time frame related to these symptoms. Patient also denies any sick family members or close contact with anyone reporting gastrointestinal symptoms. She had no history of similar episodes. Routine medications include metformin $1000 \mathrm{mg}$ tablet twice daily, gabapentin $300 \mathrm{mg}$ capsule twice daily, aspirin $81 \mathrm{mg}$ tablet daily, lisinopril $10 \mathrm{mg}$ tablet daily, albuterol nebulizers daily as needed, and levothyroxine tablet of unknown dose. Patient reports allergies to penicillin and codeine. She did not provide information regarding her reaction to these medications.

Three months prior to hospital admission, she reported starting to smoke the herb "Salvia" or Salvia divinorum, which she purchased at a local gas station. She reported smoking the herb daily for relaxation purposes. She smoked one or two cigarettes three times each day. Her current symptoms appeared approximately 48 hours after discontinuation of this product. Initially, the patient was nauseous, but symptoms progressed to diarrhea, abdominal discomfort, and vomiting over the 3 days following discontinuation.

Upon hospital admission, a physical examination and laboratory work was completed. Her blood pressure was $159 / 109 \mathrm{mmHg}$, heart rate of 76 beats per minute, respiratory rate of 20 breaths per minute, and a temperature of $97.9^{\circ}$ F. Patient appeared well-nourished and well-developed with no abnormalities found. Abdomen was tender to palpation but was not distended. She had no rebound tenderness or guarding. She was alert and oriented to person, place, and time. Patient weight was $106 \mathrm{~kg}$.

A complete blood count revealed a white blood cell count of 8,000 cells $/ \mathrm{mm}^{3}$ (normal $4.3-10,000$ cells $/ \mathrm{mm}^{3}$ ) with no other abnormalities or signs of infection. Blood chemistry revealed a fasting glucose of $118 \mathrm{mg} / \mathrm{dL}$ (normal $70-110 \mathrm{mg} / \mathrm{dL}$ ) and blood urea nitrogen level of $6 \mathrm{mg} / \mathrm{dL}$ (normal $7-18 \mathrm{mg} / \mathrm{dL}$ ). All other levels measured were within normal limits including serum creatinine of $0.91 \mathrm{mg} / \mathrm{dL}$ (normal $0.6-1.0 \mathrm{mg} / \mathrm{dL}$ ). Other laboratory tests revealed normal liver function, an $\mathrm{HbAlc}$ of $6.8 \%$, free $\mathrm{T} 4$ level of $0.76 \mathrm{ng} / \mathrm{dL}$ (normal $0.89-1.76 \mathrm{ng} / \mathrm{dL}$ ), and a TSH level of $5.9 \mu \mathrm{IU} / \mathrm{mL}(0.34-4.82)$. A fasting lipid panel revealed a total cholesterol level of $252 \mathrm{mg} / \mathrm{dL}$ (normal $<200 \mathrm{mg} / \mathrm{dL}$ ), triglyceride level of $252 \mathrm{mg} / \mathrm{dL}$ (normal 30-200 mg/dL), high density lipoprotein of $31 \mathrm{mg} / \mathrm{dL}$ (normal $35-60 \mathrm{mg} / \mathrm{dL}$ ), and a low density lipoprotein of $161 \mathrm{mg} / \mathrm{dL}$ (normal 0 $120 \mathrm{mg} / \mathrm{dL}$ ). Her amylase was $74 \mathrm{U} / \mathrm{L}$ and lipase was $100 \mathrm{U} / \mathrm{L}$, both within the normal range. A urine drug screen was positive for benzodiazepines; however, she was not currently prescribed any medications from this medication class. The drug screen was negative for opioids, marijuana, amphetamines, and cocaine. Blood cultures revealed no organism growth. Neither stool nor urine samples were taken. An arterial blood gas was not drawn.

Initial treatment included sodium chloride $0.9 \%$ i.v. fluids for dehydration, ondansetron $8 \mathrm{mg}$ i.v. every 4 hours as needed for nausea and vomiting, as well as metoclopramide $10 \mathrm{mg}$ tablet before meals and at bedtime for abdominal discomfort. Patient was kept without oral intake for one day. She remained stable overnight with no additional nausea, vomiting, or diarrhea. The following morning, she received oral intake of food and liquids without feelings of nausea or vomiting. Her white blood cell count was now at 7,000 cells $/ \mathrm{mm}^{3}$ (normal $4.3-10,000$ cells $/ \mathrm{mm}^{3}$ ). She was then discharged to continue her home medications along with ondansetron $4 \mathrm{mg}$ PO every $4-6$ hours as needed for nausea.

\section{Discussion}

The differential diagnosis included multiple factors prior to the possible diagnosis of $S$. divinorum withdrawal. First was a consideration for the possibility of a gastrointestinal virus or food poisoning. The patient reported no sick contacts at home, and she had not experienced fever. She also reported that she had not eaten outside her home within the last 48 hours and she had not eaten anything outside of her ordinary diet at home. Unfortunately stool and urine studies studies were not obtained during her hospital stay making it impossible to completely rule these out of the differential. The patient had a noncontributory past medical history and she reported no other pertinent ill contacts. Her labs including the amylase and lipase were normal. The lipid panel, triglycerides in particular, was slightly elevated but not enough to suspect pancreatitis. Urine drug screening was positive for benzodiazepines; however, it was negative for all other substances reducing the possibility of side effects or withdrawal from other common illicit medications. She did report smoking the herb S. divinorum consistently for several months. She then reported discontinuing the use of this product approximately 48 hours prior to onset of her 
reported symptoms. This led to the inclusion of withdrawal from this substance in the differential diagnosis. After close observation and treatment with i.v. fluids and antiemetics she was released from the hospital.

The active ingredient of $S$. divinorum, Salvinorin A, is a unique natural compound that is a highly potent and selective full agonist of the kappa-opioid receptor $[5,6]$. Salvinorin A is the only known substance in the Salvinorin chemical class with binding affinity at the kappaopioid receptor $[1,5]$. Salvinorin A is different from other hallucinogens such as LSD or mescaline because it has no binding affinity for the $5-\mathrm{HT}_{2 \mathrm{~A}}$ receptor $[1,3,5]$. The kappa-opioid receptor is only found in the brain and spinal cord and is commonly associated with analgesia, sedation, inhibition of gastrointestinal transit, and general dysphoria. The association between Salvinorin A stimulation of the kappa-opioid receptor and hallucinations continues to be elucidated. The lack of serotonergic properties makes this agent unique among the other hallucinogens currently popular [3]. Currently, management of Salvinorin A toxicity includes supportive care only.

The unique opioid properties may explain its ability to cause changes in intestinal transit time. A small amount of literature is available linking Salvinorin A to effects seen in the gastrointestinal (GI) system. A study by Fichna et al. looked at its effects on GI transit in mice. When given to mice, Salvinorin A inhibited cholinergic excitatory neurotransmission, reducing GI motility. This activity had antidiarrheal or constipating effects [10]. Fichna et al. continued to look into these effects on GI motility in an additional study. When Salvinorin A is given, cholinergic and noncholinergic excitatory transmission is inhibited, thus decreasing smooth muscle contraction in the GI tract [11]. Similar effects have been also seen in guinea pigs $[10,11]$. It is yet to be observed in clinical reports whether the GI effects of Salvinorin A also occur in humans with prolonged or high amounts of use; however, these animal reports do offer some explanation as to why our patient may have experienced increased GI motility and abdominal discomfort upon withdrawal of Salvinorin A.

The difficulty in establishing reproducible cause and effect with a herbal product is due to the lack of standardization amongst the Salvia divinorum products currently available. The various products may vary as much as 50 fold in potency for actual $\mathrm{mg}$ of Salvia divinorum in the product. The pharmaceutical delivery method may also impact the potency delivered to the patient. The dosage form is particularly pertinent in the case of the current patient who was smoking the herb and inhaling it [12]. The halflife of Salvia has been reported to range from 40 minutes to 1.5 hours [3]. However, these characterizations of halflife are based on single-dose pharmacokinetics. Descriptions of the physiologic behaviour of this substance after chronic use are not currently available in the literature. Variability in pharmacokinetics may explain the delayed onset of symptoms in this patient. Many of the opioid classes of agents have varied lipophilicity and the onset of withdrawal depends on half-life and the amount accumulated in fat deposits [13].

\section{Conclusion}

A 51-year-old woman developed gastrointestinal manifestations suggestive of withdrawal from Salvia divinorum after discontinuing her smoking of the substance consistently for 3 to 4 months. This may be the first documented case report of gastrointestinal symptoms of withdrawal from this substance. Considering the increased popularity of this herb, it would not be surprising to see similar reports of withdrawal in the future. Due to the lack of routine screening and the popularity of this herb, patients presenting with similar symptoms and possible risks for drug abuse should be screened for misuse of Salvia divinorum.

\section{References}

[1] M. W. Johnson, K. A. MacLean, C. J. Reissig, T. E. Prisinzano, and R. R. Griffiths, "Human psychopharmacology and doseeffects of salvinorin A, a kappa opioid agonist hallucinogen present in the plant Salvia divinorum," Drug and Alcohol Dependence, vol. 115, no. 1-2, pp. 150-155, 2011.

[2] M. J. Baggott, E. Erowid, F. Erowid, G. P. Galloway, and J. Mendelson, "Use patterns and self-reported effects of Salvia divinorum: an internet-based survey," Drug and Alcohol Dependence, vol. 111, no. 3, pp. 250-256, 2010.

[3] K. M. Babu, C. R. McCurdy, and E. W. Boyer, "Opioid receptors and legal highs: Salvia divinorum and Kratom," Clinical Toxicology, vol. 46, no. 2, pp. 146-152, 2008.

[4] M. Imanshahidi and H. Hosseinzadeh, "The pharmacological effects of Salvia species on the central nervous system," Phytotherapy Research, vol. 20, no. 6, pp. 427-437, 2006.

[5] D. González, J. Riba, J. C. Bouso, G. Gómez-Jarabo, and M. J. Barbanoj, "Pattern of use and subjective effects of Salvia divinorum among recreational users," Drug and Alcohol Dependence, vol. 85, no. 2, pp. 157-162, 2006.

[6] B. L. Roth, K. Baner, R. Westkaemper et al., "Salvinorin A: a potent naturally occurring nonnitrogenous $\kappa$ opioid selective agonist," Proceedings of the National Academy of Sciences of the United States of America, vol. 99, no. 18, pp. 11934-11939, 2002.

[7] A. A. Jenks, J. B. Walker, and S. C. Kim, "Evolution and origins of the Mazatec hallucinogenic sage, Salvia divinorum (Lamiaceae): a molecular phylogenetic approach," Journal of Plant Research, vol. 124, no. 5, pp. 593-600, 2011.

[8] P. P. Nyi, E. P. Lai, D. Y. Lee, S. A. Biglete, G. I. Torrecer, and I. B. Anderson, "Influence of age on Salvia divinorum use: results of an Internet survey," Journal of Psychoactive Drugs, vol. 42, no. 3, pp. 385-392, 2010.

[9] C. E. Dennehy, C. Tsourounis, and A. E. Miller, "Evaluation of herbal dietary supplements marketed on the internet for recreational use," Annals of Pharmacotherapy, vol. 39, no. 10, pp. 1634-1639, 2005.

[10] J. Fichna, R. Schicho, C. N. Andrews et al., "Salvinorin A inhibits colonic transit and neurogenic ion transport in mice by activating $\kappa$-opioid and cannabinoid receptors," $\mathrm{Neu}$ rogastroenterology and Motility, vol. 21, no. 12, pp. 1326-e127, 2009.

[11] J. Fichna, R. Schicho, A. Janecka, J. K. Zjawiony, and M. Storr, "Selective natural $\kappa$ opioid and cannabinoid receptor agonists with a potential role in the treatment of gastrointestinal dysfunction," Drug News and Perspectives, vol. 22, no. 7, pp. 383$392,2009$. 
[12] R. Vohra, A. Seefeld, F. L. Cantrell, and R. F. Clark, "Salvia divinorum: exposures reported to a statewide poison control system over 10 years," Journal of Emergency Medicine, vol. 40, no. 6, pp. 643-650, 2011.

[13] H. B. Gutstein and H. Akil, "Opioid analgesics," in The Pharmacological Basis of Therapeutics, L. L. Brunton, J. S. Lazo, and K. L. Parker, Eds., pp. 547-590, McGraw Hill, New York, NY, USA, 11th edition, 2006. 


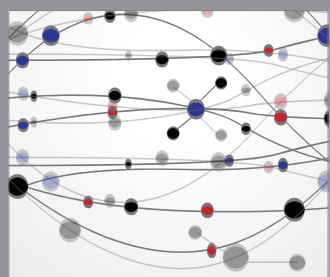

The Scientific World Journal
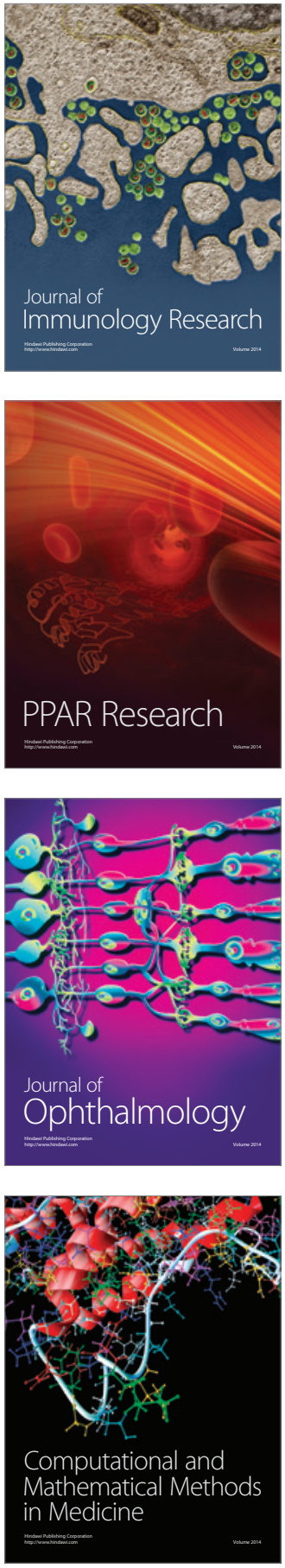

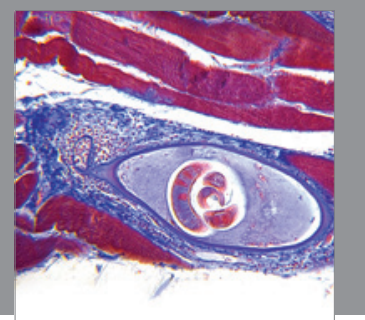

Gastroenterology

Research and Practice
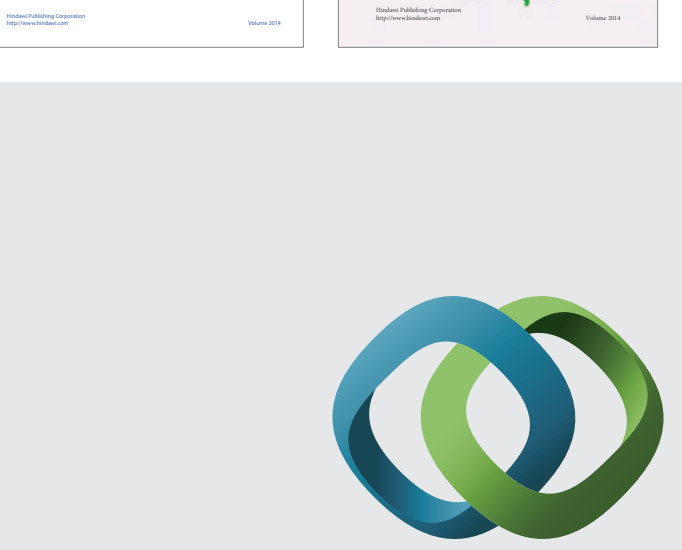

\section{Hindawi}

Submit your manuscripts at

http://www.hindawi.com
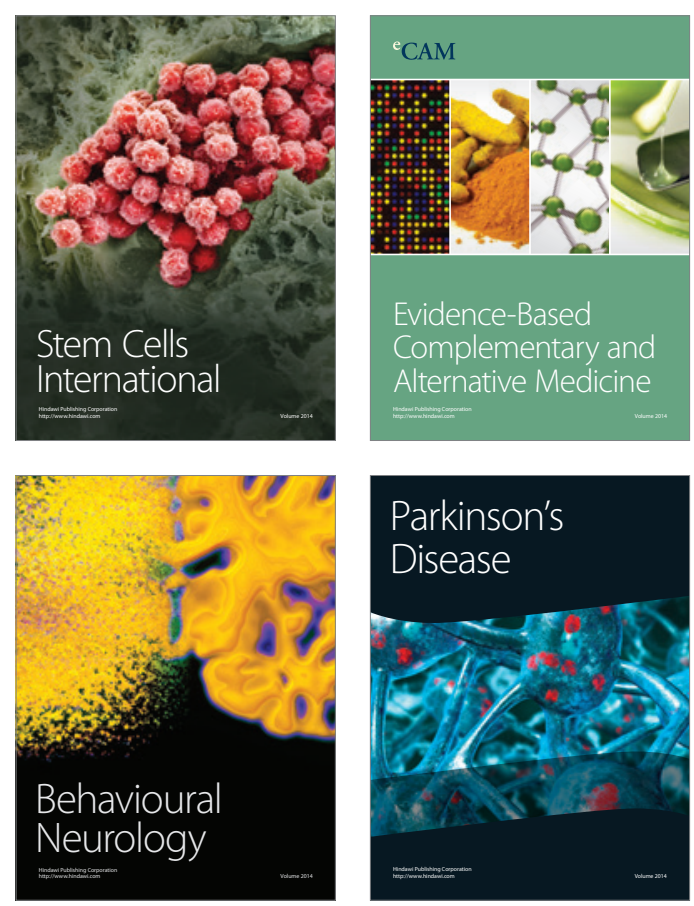

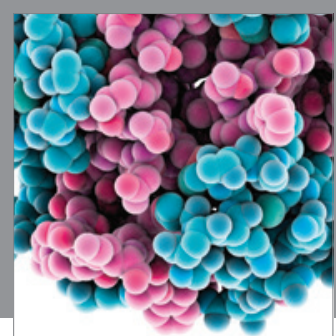

Journal of
Diabetes Research

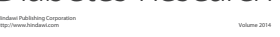

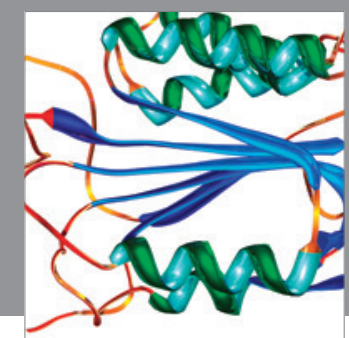

Disease Markers
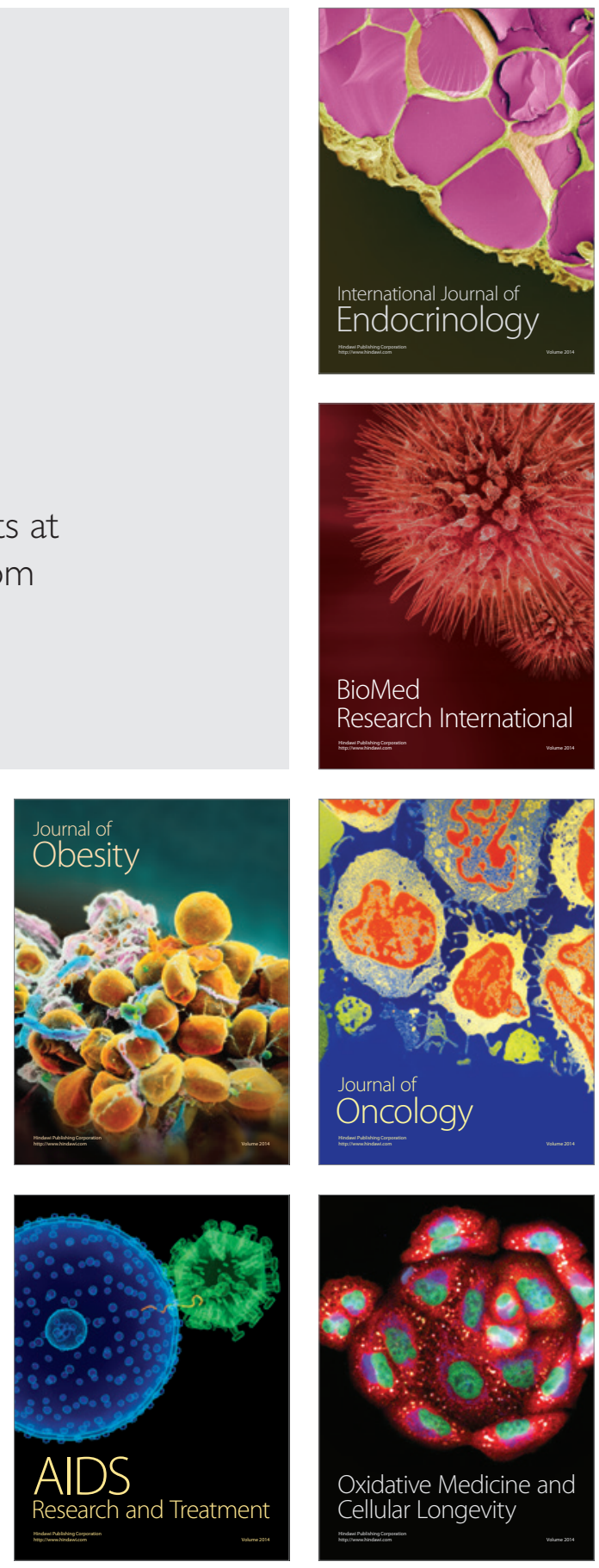